\title{
Ostrowski type inequalities involving conformable fractional integrals
}

\author{
Muhammad Adil Khan ${ }^{1,2}$, Sumbel Begum², Yousaf Khurshid ${ }^{2}$ and Yu-Ming Chu ${ }^{3 *}$
}

"Correspondence:

chuyuming2005@126.com

${ }^{3}$ Department of Mathematics, Huzhou University, Huzhou, China

Full list of author information is

available at the end of the article

\begin{abstract}
In the article, we establish several Ostrowski type inequalities involving the conformable fractional integrals. As applications, we find new inequalities for the arithmetic and generalized logarithmic means.
\end{abstract}

MSC: 26D15; 26A51; 26A33; 26E60

Keywords: Ostrowski inequality; Conformable derivative; Conformable integral; Arithmetic mean; Generalized logarithmic mean

\section{Introduction}

Let $I \subseteq \mathbb{R}$ be an interval and $I^{\circ}$ the interior of $I$. Then the classical Ostrowski inequality [1] states that a real-valued function $f: I \rightarrow \mathbb{R}$ satisfies the inequality

$$
\left|f(x)-\frac{1}{a_{2}-a_{1}} \int_{a_{1}}^{a_{2}} f(x) d x\right| \leq\left[\frac{1}{4}+\frac{\left(x-\frac{a_{1}+a_{2}}{2}\right)^{2}}{\left(a_{2}-a_{1}\right)^{2}}\right]\left(a_{2}-a_{1}\right)\left\|f^{\prime}\right\|_{\infty}
$$

with the best possible constant $1 / 4$ if $a_{1}, a_{2} \in I^{\circ}$ with $a_{1}<a_{2}$ and $\left|f^{\prime}(x)\right| \leq M$ for all $x \in$ $\left[a_{1}, a_{2}\right]$.

Recently, the Ostrowski inequality has attracted the attention of many researchers, many remarkable generalizations, extensions, variants and applications can be found in the literature [2-24].

Let $0<\alpha \leq 1$ and $g$ be a real-valued function defined on $[0, \infty)$. Then the (conformable) fractional derivative $D_{\alpha}(g)(t)$ [23] of order $\alpha$ of $g$ at $t>0$ is defined by

$$
D_{\alpha}(g)(t)=\lim _{\epsilon \rightarrow 0} \frac{g\left(t+\epsilon t^{1-\alpha}\right)-g(t)}{\epsilon} .
$$

$g$ is said to be $\alpha$-differentiable if the conformable fractional derivative of order $\alpha$ of $g$ exists. In what follows, we write $g^{\alpha}(t)$ or $\frac{d_{\alpha}}{d_{\alpha} t}(g)$ for $D_{\alpha}(g)(t)$ to denote the conformable fractional derivative of order $\alpha$ of $g$. The conformable fractional derivative at 0 is defined as $g^{\alpha}(0)=\lim _{t \rightarrow 0^{+}} g^{\alpha}(t)$.

Let $\alpha \in(0,1]$ and $0 \leq a<b$. Then the function $h:[a, b] \rightarrow \mathbb{R}$ is said to be $\alpha$-fractional integrable on $[a, b]$ if the integral

$$
\int_{a}^{b} h(x) d_{\alpha} x:=\int_{a}^{b} h(x) x^{\alpha-1} d x
$$

(c) The Author(s) 2018. This article is distributed under the terms of the Creative Commons Attribution 4.0 International License (http://creativecommons.org/licenses/by/4.0/), which permits unrestricted use, distribution, and reproduction in any medium, provided you give appropriate credit to the original author(s) and the source, provide a link to the Creative Commons license, and indicate if changes were made. 
exists and is finite. All $\alpha$-fractional integrable functions on $[a, b]$ are denoted by $L_{\alpha}^{1}([a, b])$.

Remark 1.1 Note that the relation between the Riemann integral and the conformable fractional integral is given by

$$
I_{\alpha}^{a}(h)(t)=I_{1}^{a}\left(t^{\alpha-1} h\right)=\int_{a}^{t} \frac{h(x)}{x^{1-\alpha}} d x .
$$

Let $\alpha \in(0,1]$ and $f, g$ be $\alpha$-differentiable at $t>0$. Then it is well known that

$$
\text { (1) } \frac{d_{\alpha}}{d_{\alpha} t}\left(t^{n}\right)=n t^{n-\alpha}
$$

for all $n \in \mathbb{R}$;

$$
\text { (2) } \frac{d_{\alpha}}{d_{\alpha} t}(c)=0
$$

for all constant $c \in \mathbb{R}$;

$$
\text { (3) } \frac{d_{\alpha}}{d_{\alpha} t}(a f(t)+b g(t))=a \frac{d_{\alpha}}{d_{\alpha} t}(f(t))+b \frac{d_{\alpha}}{d_{\alpha} t}(g(t))
$$

for all $a, b \in \mathbb{R}$;

(4) $\frac{d_{\alpha}}{d_{\alpha} t}(f(t) g(t))=f(t) \frac{d_{\alpha}}{d_{\alpha} t}(g(t))+g(t) \frac{d_{\alpha}}{d_{\alpha} t}(f(t))$;

(5) $\frac{d_{\alpha}}{d_{\alpha} t}\left(\frac{f(t)}{g(t)}\right)=\frac{g(t) \frac{d_{\alpha}}{d_{\alpha} t}(f(t))-f(t) \frac{d_{\alpha}}{d_{\alpha} t}(g(t))}{g^{2}(t)}$;

(6) $\frac{d_{\alpha}}{d_{\alpha} t}(f(g(t)))=f^{\prime}(g(t)) \frac{d_{\alpha}}{d_{\alpha} t}(g(t))$,

if $f$ is differentiable at $g(t)$.

The main purpose of the article is to find the Ostrowski type inequalities involving the conformable fractional integrals and give their applications in certain bivariate means.

\section{Main results}

Lemma 2.1 Let $0<\alpha \leq 1,0 \leq a_{1}<a_{2}$ and $h:\left[a_{1}, a_{2}\right] \rightarrow \mathbb{R}$ be an $\alpha$-fractional differentiable function. Then the identity

$$
\begin{aligned}
h(x)-\frac{\alpha}{a_{2}^{\alpha}-a_{1}^{\alpha}} \int_{a_{1}}^{a_{2}} h(s) d_{\alpha} s= & \frac{x-a_{1}}{a_{2}^{\alpha}-a_{1}^{\alpha}} \int_{0}^{1}\left[\left((1-t) a_{1}+t x\right)^{2 \alpha-1}-a_{1}^{\alpha}\left((1-t) a_{1}+t x\right)^{\alpha-1}\right] \\
& \times D_{\alpha}(h)\left((1-t) a_{1}+t x\right) t^{1-\alpha} d_{\alpha} t \\
& +\frac{a_{2}-x}{a_{2}^{\alpha}-a_{1}^{\alpha}} \int_{0}^{1}\left[\left((1-t) a_{2}+t x\right)^{2 \alpha-1}-a_{2}^{\alpha}\left((1-t) a_{2}+t x\right)^{\alpha-1}\right] \\
& \times D_{\alpha}(h)\left((1-t) a_{2}+t x\right) t^{1-\alpha} d_{\alpha} t
\end{aligned}
$$

holds if $D_{\alpha}(h) \in L_{\alpha}^{1}\left(\left[a_{1}, a_{2}\right]\right)$. 
Proof It follows from integration by parts that

$$
\begin{aligned}
& \frac{x-a_{1}}{a_{2}^{\alpha}-a_{1}^{\alpha}} \int_{0}^{1}\left[\left((1-t) a_{1}+t x\right)^{2 \alpha-1}-a_{1}^{\alpha}\left((1-t) a_{1}+t x\right)^{\alpha-1}\right] D_{\alpha}(h)\left((1-t) a_{1}+t x\right) t^{1-\alpha} d_{\alpha} t \\
& +\frac{a_{2}-x}{a_{2}^{\alpha}-a_{1}^{\alpha}} \int_{0}^{1}\left[\left((1-t) a_{2}+t x\right)^{2 \alpha-1}-a_{2}^{\alpha}\left((1-t) a_{2}+t x\right)^{\alpha-1}\right] \\
& \times D_{\alpha}(h)\left((1-t) a_{2}+t x\right) t^{1-\alpha} d_{\alpha} t \\
& =\frac{x-a_{1}}{a_{2}^{\alpha}-a_{1}^{\alpha}} \int_{0}^{1}\left[\left((1-t) a_{1}+t x\right)^{2 \alpha-1}-a_{1}^{\alpha}\left((1-t) a_{1}+t x\right)^{\alpha-1}\right] D_{\alpha}(h)\left((1-t) a_{1}+t x\right) d t \\
& +\frac{a_{2}-x}{a_{2}^{\alpha}-a_{1}^{\alpha}} \int_{0}^{1}\left[\left((1-t) a_{2}+t x\right)^{2 \alpha-1}-a_{2}^{\alpha}\left((1-t) a_{2}+t x\right)^{\alpha-1}\right] \\
& \times D_{\alpha}(h)\left((1-t) a_{2}+t x\right) d t \\
& =\frac{x-a_{1}}{a_{2}^{\alpha}-a_{1}^{\alpha}} \int_{0}^{1}\left[\left((1-t) a_{1}+t x\right)^{\alpha}-a_{1}^{\alpha}\right] h^{\prime}\left((1-t) a_{1}+t x\right) d t \\
& +\frac{a_{2}-x}{a_{2}^{\alpha}-a_{1}^{\alpha}} \int_{0}^{1}\left[\left((1-t) a_{2}+t x\right)^{\alpha}-a_{2}^{\alpha}\right] h^{\prime}\left((1-t) a_{2}+t x\right) d t \\
& =\left.\frac{x-a_{1}}{a_{2}^{\alpha}-a_{1}^{\alpha}}\left(\left((1-t) a_{1}+t x\right)^{\alpha}-a_{1}^{\alpha}\right) \frac{h\left((1-t) a_{1}+t x\right)}{x-a_{1}}\right|_{0} ^{1} \\
& -\frac{x-a_{1}}{a_{2}^{\alpha}-a_{1}^{\alpha}} \int_{0}^{1} \alpha\left((1-t) a_{1}+t x\right)^{\alpha-1} h\left((1-t) a_{1}+t x\right) d t \\
& +\left.\frac{a_{2}-x}{a_{2}^{\alpha}-a_{1}^{\alpha}}\left(\left((1-t) a_{2}+t x\right)^{\alpha}-a_{2}^{\alpha}\right) \frac{h\left((1-t) a_{2}+t x\right)}{x-a_{2}}\right|_{0} ^{1} \\
& -\frac{a_{2}-x}{a_{2}^{\alpha}-a_{1}^{\alpha}} \int_{0}^{1} \alpha\left((1-t) a_{2}+t x\right)^{\alpha-1} h\left((1-t) a_{2}+t x\right) d t \\
& =\frac{x-a_{1}}{a_{2}^{\alpha}-a_{1}^{\alpha}}\left(\frac{x^{\alpha}-a_{1}^{\alpha}}{x-a_{1}} h(x)-\frac{\alpha}{x-a_{1}} \int_{a_{1}}^{x} h(s) d_{\alpha} s\right) \\
& +\frac{a_{2}-x}{a_{2}^{\alpha}-a_{1}^{\alpha}}\left(\frac{a_{2}^{\alpha}-x^{\alpha}}{a_{2}-x} h(x)-\frac{\alpha}{a_{2}-x} \int_{x}^{a_{2}} h(s) d_{\alpha} s\right) \\
& =h(x)-\frac{\alpha}{a_{2}^{\alpha}-a_{1}^{\alpha}} \int_{a_{1}}^{a_{2}} h(s) d_{\alpha} s \text {. }
\end{aligned}
$$

Theorem 2.2 Let $0<\alpha \leq 1,0 \leq a_{1}<a_{2}, h:\left[a_{1}, a_{2}\right] \rightarrow \mathbb{R}$ be an $\alpha$-fractional differentiable function and $D_{\alpha}(h) \in L_{\alpha}^{1}\left(\left[a_{1}, a_{2}\right]\right)$. Then the inequality

$$
\left|h(x)-\frac{\alpha}{a_{2}^{\alpha}-a_{1}^{\alpha}} \int_{a_{1}}^{a_{2}} h(s) d_{\alpha} s\right| \leq \frac{x-a_{1}}{a_{2}^{\alpha}-a_{1}^{\alpha}} \Delta_{1}+\frac{a_{2}-x}{a_{2}^{\alpha}-a_{1}^{\alpha}} \triangle_{2}
$$

holds if $\left|h^{\prime}(x)\right|$ is convex, where

$$
\begin{aligned}
\triangle_{1}= & \frac{1}{6} a_{1}^{\alpha-1} x\left|h^{\prime}\left(a_{1}\right)\right|+\frac{1}{12} x^{\alpha-1} a_{1}\left|h^{\prime}\left(a_{1}\right)\right|+\frac{1}{12} x\left|h^{\prime}\left(a_{1}\right)\right|-\frac{1}{4} a_{1}^{\alpha}\left|h^{\prime}\left(a_{1}\right)\right| \\
& +\frac{1}{12} a_{1}\left|h^{\prime}(x)\right|+\frac{1}{12} x^{\alpha-1} a_{1}\left|h^{\prime}(x)\right|+\frac{1}{4} x\left|h^{\prime}(x)\right|-\frac{1}{2} a_{1}^{\alpha}\left|h^{\prime}(x)\right|, \\
\triangle_{2}= & \frac{1}{6} a_{2}^{\alpha}\left|h^{\prime}\left(a_{2}\right)\right|-\frac{1}{6} x^{\alpha}\left|h^{\prime}\left(a_{2}\right)\right|+\frac{1}{3} a_{2}^{\alpha}\left|h^{\prime}(x)\right|-\frac{1}{3} x^{\alpha}\left|h^{\prime}(x)\right| .
\end{aligned}
$$


Proof Let $y>0, \varphi_{1}(y)=y^{\alpha-1}$ and $\varphi_{2}(y)=-y^{\alpha}$. Then we clearly see that the functions $\varphi_{1}$ and $\varphi_{2}$ both are convex. It follows from Lemma 2.1 and the convexity of $\varphi_{1}, \varphi_{2}$ and $\left|h^{\prime}\right|$ that

$$
\begin{aligned}
& \left|h(x)-\frac{\alpha}{a_{2}^{\alpha}-a_{1}^{\alpha}} \int_{a_{1}}^{a_{2}} h(s) d_{\alpha} s\right| \\
& \leq \frac{x-a_{1}}{a_{2}^{\alpha}-a_{1}^{\alpha}} \int_{0}^{1}\left(\left((1-t) a_{1}+t x\right)^{\alpha}-a_{1}^{\alpha}\right)\left|h^{\prime}\left((1-t) a_{1}+t x\right)\right| d t \\
& +\frac{a_{2}-x}{a_{2}^{\alpha}-a_{1}^{\alpha}} \int_{0}^{1}\left(a_{2}^{\alpha}-\left((1-t) a_{2}+t x\right)^{\alpha}\right)\left|h^{\prime}\left((1-t) a_{2}+t x\right)\right| d t \\
& \leq \frac{x-a_{1}}{a_{2}^{\alpha}-a_{1}^{\alpha}} \int_{0}^{1}\left(\left((1-t) a_{1}+t x\right)^{\alpha-1}\left((1-t) a_{1}+t x\right)-a_{1}^{\alpha}\right)\left|h^{\prime}\left((1-t) a_{1}+t x\right)\right| d t \\
& +\frac{a_{2}-x}{a_{2}^{\alpha}-a_{1}^{\alpha}} \int_{0}^{1}\left(a_{2}^{\alpha}-\left((1-t) a_{2}^{\alpha}+t x^{\alpha}\right)\right)\left|h^{\prime}\left((1-t) a_{2}+t x\right)\right| d t \\
& \leq \frac{x-a_{1}}{a_{2}^{\alpha}-a_{1}^{\alpha}} \int_{0}^{1}\left(\left((1-t) a_{1}^{\alpha-1}+t x^{\alpha-1}\right)\left((1-t) a_{1}+t x\right)-a_{1}^{\alpha}\right)\left|h^{\prime}\left((1-t) a_{1}+t x\right)\right| d t \\
& +\frac{a_{2}-x}{a_{2}^{\alpha}-a_{1}^{\alpha}} \int_{0}^{1}\left(a_{2}^{\alpha}-\left((1-t) a_{2}^{\alpha}+t x^{\alpha}\right)\right)\left|h^{\prime}\left((1-t) a_{2}+t x\right)\right| d t \\
& \leq \frac{x-a_{1}}{a_{2}^{\alpha}-a_{1}^{\alpha}} \int_{0}^{1}\left(\left((1-t) a_{1}^{\alpha-1}+t x^{\alpha-1}\right)\left((1-t) a_{1}+t x\right)-a_{1}^{\alpha}\right) \\
& \times\left[(1-t)\left|h^{\prime}\left(a_{1}\right)\right|+t\left|h^{\prime}(x)\right|\right] d t \\
& +\frac{a_{2}-x}{a_{2}^{\alpha}-a_{1}^{\alpha}} \int_{0}^{1}\left(a_{2}^{\alpha}-\left((1-t) a_{2}^{\alpha}+t x^{\alpha}\right)\right)\left[(1-t)\left|h^{\prime}\left(a_{2}\right)\right|+t\left|h^{\prime}(x)\right|\right] d t \\
& =\frac{x-a_{1}}{a_{2}^{\alpha}-a_{1}^{\alpha}} \Delta_{1}+\frac{a_{2}-x}{a_{2}^{\alpha}-a_{1}^{\alpha}} \Delta_{2} \text {. }
\end{aligned}
$$

Corollary 2.3 Let $x=\left(a_{1}+a_{2}\right) / 2$. Then Theorem 2.2 leads to

$$
\begin{aligned}
\left|h\left(\frac{a_{1}+a_{2}}{2}\right)-\frac{\alpha}{a_{2}^{\alpha}-a_{1}^{\alpha}} \int_{a_{1}}^{a_{2}} h(s) d_{\alpha} s\right| \\
\leq \frac{a_{2}-a_{1}}{2\left(a_{2}^{\alpha}-a_{1}^{\alpha}\right)}\left[\left(\frac{2 a_{1}^{\alpha-1} a_{2}-10 a_{1}^{\alpha}+a_{1}+a_{2}}{24}\right)\left|h^{\prime}\left(a_{1}\right)\right|+\frac{a_{1}}{12}\left(\frac{a_{1}+a_{2}}{2}\right)^{\alpha-1}\left|h^{\prime}\left(a_{1}\right)\right|\right. \\
\quad+\left(\frac{5 a_{1}+3 a_{2}-12 a_{1}^{\alpha}}{24}\right)\left|h^{\prime}\left(\frac{a_{1}+a_{2}}{2}\right)\right|+\frac{a_{1}}{12}\left(\frac{a_{1}+a_{2}}{2}\right)^{\alpha-1}\left|h^{\prime}\left(\frac{a_{1}+a_{2}}{2}\right)\right| \\
\quad+\frac{1}{6} a_{2}^{\alpha}\left|h^{\prime}\left(a_{2}\right)\right|-\frac{1}{6}\left(\frac{a_{1}+a_{2}}{2}\right)^{\alpha}\left|h^{\prime}\left(a_{2}\right)\right| \\
\left.\quad+\frac{a_{2}^{\alpha}}{3}\left|h^{\prime}\left(\frac{a_{1}+a_{2}}{2}\right)\right|-\frac{1}{3}\left(\frac{a_{1}+a_{2}}{2}\right)^{\alpha}\left|h^{\prime}\left(\frac{a_{1}+a_{2}}{2}\right)\right|\right] .
\end{aligned}
$$

Remark 2.4 If $\alpha=1$, then Corollary 2.3 becomes

$$
\begin{aligned}
\left|h\left(\frac{a_{1}+a_{2}}{2}\right)-\frac{\alpha}{a_{2}^{\alpha}-a_{1}^{\alpha}} \int_{a_{1}}^{a_{2}} h(s) d_{\alpha} s\right| & \leq \frac{a_{2}-a_{1}}{24}\left(\left|h^{\prime}\left(a_{1}\right)\right|+4\left|h^{\prime}\left(\frac{a_{1}+a_{2}}{2}\right)\right|+\left|h^{\prime}\left(a_{2}\right)\right|\right) \\
& \leq \frac{a_{2}-a_{1}}{8}\left(\left|h^{\prime}\left(a_{1}\right)\right|+\left|h^{\prime}\left(a_{2}\right)\right|\right),
\end{aligned}
$$

where the second inequality is obtained by using the convexity of $\left|h^{\prime}\right|$. 
Theorem 2.5 Let $q>1, M>0,0<\alpha \leq 1,0 \leq a_{1}<a_{2}, h:\left[a_{1}, a_{2}\right] \rightarrow \mathbb{R}$ be an $\alpha$-fractional differentiable function and $D_{\alpha}(h) \in L_{\alpha}^{1}\left(\left[a_{1}, a_{2}\right]\right)$. Then the inequality

$$
\begin{aligned}
& \left|h(x)-\frac{\alpha}{a_{2}^{\alpha}-a_{1}^{\alpha}} \int_{a_{1}}^{a_{2}} h(s) d_{\alpha} s\right| \\
& \leq M \frac{x-a_{1}}{a_{2}^{\alpha}-a_{1}^{\alpha}}\left(A_{1}(\alpha)\right)^{1-1 / q}\left(A_{2}(\alpha)+A_{3}(\alpha)\right)^{1 / q} \\
& \quad+M \frac{a_{2}-x}{a_{2}^{\alpha}-a_{1}^{\alpha}}\left(B_{1}(\alpha)\right)^{1-1 / q}\left(B_{2}(\alpha)+B_{3}(\alpha)\right)^{1 / q}
\end{aligned}
$$

holds if $\left|h^{\prime}\right|^{q}$ is convex on $\left[a_{1}, a_{2}\right]$ and $\left|h^{\prime}(x)\right|^{q} \leq M$, where

$$
\begin{aligned}
& A_{1}(\alpha)=\frac{x^{\alpha+1}-a_{1}^{\alpha+1}}{(\alpha+1)\left(x-a_{1}\right)}-a_{1}^{\alpha}, \quad B_{1}(\alpha)=a_{2}^{\alpha}-\frac{x^{\alpha+1}-a_{2}^{\alpha+1}}{(\alpha+1)\left(a_{2}-x\right)}, \\
& A_{2}(\alpha)=-\frac{a_{1}^{\alpha+1}}{(\alpha+1)\left(x-a_{1}\right)} \frac{(\alpha+2)\left(x-a_{1}\right)+a_{1}}{(\alpha+2)\left(x-a_{1}\right)}+\frac{x^{\alpha+2}}{(\alpha+1)\left(x-a_{1}\right)^{2}(\alpha+2)}-\frac{a_{1}^{\alpha}}{2}, \\
& B_{2}(\alpha)=\frac{a_{2}^{\alpha}}{2}+\frac{a_{2}^{\alpha+1}}{(\alpha+1)\left(a_{2}-x\right)} \frac{(\alpha+2)\left(a_{2}-x\right)+a_{2}}{(\alpha+2)\left(a_{2}-x\right)}-\frac{x^{\alpha+2}}{(\alpha+1)\left(a_{2}-x\right)^{2}(\alpha+2)}, \\
& A_{3}(\alpha)=\frac{x^{\alpha+1}}{(\alpha+1)\left(x-a_{1}\right)} \frac{(\alpha+2)\left(x-a_{1}\right)-x}{(\alpha+2)\left(x-a_{1}\right)}+\frac{a_{1}^{\alpha+2}}{(\alpha+1)\left(x-a_{1}\right)^{2}(\alpha+2)}-\frac{a_{1}^{\alpha}}{2}, \\
& B_{3}(\alpha)=\frac{a_{2}^{\alpha}}{2}-\frac{x^{\alpha+1}}{(\alpha+1)\left(a_{2}-x\right)} \frac{(\alpha+2)\left(a_{2}-x\right)-x}{(\alpha+2)\left(a_{2}-x\right)}-\frac{a_{2}^{\alpha+2}}{(\alpha+1)\left(a_{2}-x\right)^{2}(\alpha+2)} .
\end{aligned}
$$

Proof From Lemma 2.1, power-mean inequality and the convexity of $\left|h^{\prime}\right|^{q}$ together with the identities

$$
\int_{0}^{1}\left(\left((1-t) a_{1}+t x\right)^{\alpha}-a_{1}^{\alpha}\right) d t=\frac{x^{\alpha+1}-a_{1}^{\alpha+1}}{(\alpha+1)\left(x-a_{1}\right)}-a_{1}^{\alpha}
$$

and

$$
\int_{0}^{1}\left(a_{2}^{\alpha}-\left((1-t) a_{2}+t x\right)^{\alpha}\right) d t=a_{2}^{\alpha}-\frac{x^{\alpha+1}-a_{2}^{\alpha+1}}{(\alpha+1)\left(a_{2}-x\right)}
$$

we clearly see that

$$
\begin{aligned}
& \left|h(x)-\frac{\alpha}{a_{2}^{\alpha}-a_{1}^{\alpha}} \int_{a_{1}}^{a_{2}} h(s) d_{\alpha} s\right| \\
& \leq \frac{x-a_{1}}{a_{2}^{\alpha}-a_{1}^{\alpha}} \int_{0}^{1}\left(\left((1-t) a_{1}+t x\right)^{\alpha}-a_{1}^{\alpha}\right)\left|h^{\prime}\left((1-t) a_{1}+t x\right)\right| d t \\
& \quad+\frac{a_{2}-x}{a_{2}^{\alpha}-a_{1}^{\alpha}} \int_{0}^{1}\left(a_{2}^{\alpha}-\left((1-t) a_{2}+t x\right)^{\alpha}\right)\left|h^{\prime}\left((1-t) a_{2}+t x\right)\right| d t \\
& \int_{0}^{1}\left(\left((1-t) a_{1}+t x\right)^{\alpha}-a_{1}^{\alpha}\right)\left|h^{\prime}\left((1-t) a_{1}+t x\right)\right| d t \\
& \leq\left(\int_{0}^{1}\left(\left((1-t) a_{1}+t x\right)^{\alpha}-a_{1}^{\alpha}\right) d t\right)^{1-1 / q} \\
& \quad \times\left(\int_{0}^{1}\left(\left((1-t) a_{1}+t x\right)^{\alpha}-a_{1}^{\alpha}\right)\left|h^{\prime}\left((1-t) a_{1}+t x\right)\right|^{q} d t\right)^{1 / q},
\end{aligned}
$$




$$
\begin{aligned}
& \int_{0}^{1}\left(a_{2}^{\alpha}-\left((1-t) a_{2}+t x\right)^{\alpha}\right)\left|h^{\prime}\left((1-t) a_{2}+t x\right)\right| d t \\
& \leq\left(\int_{0}^{1}\left(a_{2}^{\alpha}-\left((1-t) a_{2}+t x\right)^{\alpha}\right) d t\right)^{1-1 / q} \\
& \times\left(\int_{0}^{1}\left(a_{2}^{\alpha}-\left((1-t) a_{2}+t x\right)^{\alpha}\right)\left|h^{\prime}\left((1-t) a_{2}+t x\right)\right|^{q} d t\right)^{1 / q}, \\
& \int_{0}^{1}\left(\left((1-t) a_{1}+t x\right)^{\alpha}-a_{1}^{\alpha}\right)\left|h^{\prime}\left((1-t) a_{1}+t x\right)\right|^{q} d t \\
& \leq \int_{0}^{1}\left(\left((1-t) a_{1}+t x\right)^{\alpha}-a_{1}^{\alpha}\right)\left[(1-t)\left|h^{\prime}\left(a_{1}\right)\right|^{q}+t\left|h^{\prime}(x)\right|^{q}\right] d t \\
& =\left|h^{\prime}(a)\right|^{q} \int_{0}^{1}\left(\left((1-t) a_{1}+t x\right)^{\alpha}-a_{1}^{\alpha}\right)(1-t) d t+\left|h^{\prime}(x)\right|^{q} \int_{0}^{1}\left(\left((1-t) a_{1}+t x\right)^{\alpha}-a_{1}^{\alpha}\right) t d t \\
& =\left|h^{\prime}(a)\right|^{q}\left(-\frac{a_{1}^{\alpha+1}}{(\alpha+1)\left(x-a_{1}\right)} \frac{(\alpha+2)\left(x-a_{1}\right)+a_{1}}{(\alpha+2)\left(x-a_{1}\right)}+\frac{x^{\alpha+2}}{(\alpha+1)\left(x-a_{1}\right)^{2}(\alpha+2)}-\frac{a_{1}^{\alpha}}{2}\right) \\
& +\left|h^{\prime}(x)\right|^{q}\left(\frac{x^{\alpha+1}}{(\alpha+1)\left(x-a_{1}\right)} \frac{(\alpha+2)\left(x-a_{1}\right)-x}{(\alpha+2)\left(x-a_{1}\right)}+\frac{a_{1}^{\alpha+2}}{(\alpha+1)\left(x-a_{1}\right)^{2}(\alpha+2)}-\frac{a_{1}^{\alpha}}{2}\right) \\
& \leq M^{q}\left(-\frac{a_{1}^{\alpha+1}}{(\alpha+1)\left(x-a_{1}\right)} \frac{(\alpha+2)\left(x-a_{1}\right)+a_{1}}{(\alpha+2)\left(x-a_{1}\right)}+\frac{x^{\alpha+2}}{(\alpha+1)\left(x-a_{1}\right)^{2}(\alpha+2)}-\frac{a_{1}^{\alpha}}{2}\right) \\
& +M^{q}\left(\frac{x^{\alpha+1}}{(\alpha+1)\left(x-a_{1}\right)} \frac{(\alpha+2)\left(x-a_{1}\right)-x}{(\alpha+2)\left(x-a_{1}\right)}+\frac{a_{1}^{\alpha+2}}{(\alpha+1)\left(x-a_{1}\right)^{2}(\alpha+2)}-\frac{a_{1}^{\alpha}}{2}\right), \\
& \int_{0}^{1}\left(a_{2}^{\alpha}-\left((1-t) a_{2}+t x\right)^{\alpha}\right)\left|h^{\prime}\left((1-t) a_{2}+t x\right)\right|^{q} d t \\
& \leq \int_{0}^{1}\left(a_{2}^{\alpha}-\left((1-t) a_{2}+t x\right)^{\alpha}\right)\left[(1-t)\left|h^{\prime}\left(a_{2}\right)\right|^{q}+t\left|h^{\prime}(x)\right|^{q}\right] d t \\
& =\left|h^{\prime}\left(a_{2}\right)\right|^{q} \int_{0}^{1}\left(a_{2}^{\alpha}-\left((1-t) a_{2}+t x\right)^{\alpha}\right)(1-t) d t \\
& +\left|h^{\prime}(x)\right|^{q} \int_{0}^{1}\left(a_{2}^{\alpha}-\left((1-t) a_{2}+t x\right)^{\alpha}\right) t d t \\
& =\left|h^{\prime}\left(a_{2}\right)\right|^{q}\left(\frac{a_{2}^{\alpha}}{2}+\frac{a_{2}^{\alpha+1}}{(\alpha+1)\left(a_{2}-x\right)} \frac{(\alpha+2)\left(a_{2}-x\right)+a_{2}}{(\alpha+2)\left(a_{2}-x\right)}-\frac{x^{\alpha+2}}{(\alpha+1)\left(a_{2}-x\right)^{2}(\alpha+2)}\right) \\
& +\left|h^{\prime}(x)\right|^{q}\left(\frac{a_{2}^{\alpha}}{2}-\frac{x^{\alpha+1}}{(\alpha+1)\left(a_{2}-x\right)} \frac{(\alpha+2)\left(a_{2}-x\right)-x}{(\alpha+2)\left(a_{2}-x\right)}-\frac{a_{2}^{\alpha+2}}{(\alpha+1)\left(a_{2}-x\right)^{2}(\alpha+2)}\right) \\
& \leq M^{q}\left(\frac{a_{2}^{\alpha}}{2}+\frac{a_{2}^{\alpha+1}}{(\alpha+1)\left(a_{2}-x\right)} \frac{(\alpha+2)\left(a_{2}-x\right)+a_{2}}{(\alpha+2)\left(a_{2}-x\right)}-\frac{x^{\alpha+2}}{(\alpha+1)\left(a_{2}-x\right)^{2}(\alpha+2)}\right) \\
& +M^{q}\left(\frac{a_{2}^{\alpha}}{2}-\frac{x^{\alpha+1}}{(\alpha+1)\left(a_{2}-x\right)} \frac{(\alpha+2)\left(a_{2}-x\right)-x}{(\alpha+2)\left(a_{2}-x\right)}-\frac{a_{2}^{\alpha+2}}{(\alpha+1)\left(a_{2}-x\right)^{2}(\alpha+2)}\right) .
\end{aligned}
$$

Therefore, Theorem 2.5 follows easily from (2.1)-(2.5).

Remark 2.6 Let $\alpha=1$. Then Theorem 2.5 leads to

$$
\begin{aligned}
\left|h(x)-\frac{1}{a_{2}-a_{1}} \int_{a_{1}}^{a_{2}} h(s) d s\right| \leq & M \frac{x-a_{1}}{a_{2}-a_{1}}\left(A_{1}(1)\right)^{1-1 / q}\left[A_{2}(1)+A_{3}(1)\right]^{1 / q} \\
& +M \frac{a_{2}-x}{a_{2}-a_{1}}\left(B_{1}(1)\right)^{1-1 / q}\left[B_{2}(1)+B_{3}(1)\right]^{1 / q}
\end{aligned}
$$


where

$$
\begin{aligned}
& A_{1}(1)=\frac{x-a_{1}}{2}, \quad B_{1}(1)=\frac{a_{2}-x}{2}, \\
& A_{2}(1)=\frac{3 a_{1}^{2} x+6 a_{1}^{2}+x^{3}-3 a_{1} x^{2}-3 a_{1}^{3}}{6\left(x-a_{1}\right)^{2}}, \quad B_{2}(1)=\frac{7 a_{2}^{3}+3 a_{2} x^{2}-9 a_{2}^{2} x-x^{3}}{6\left(a_{2}-x\right)^{2}}, \\
& A_{3}(1)=\frac{2 x^{3}-2 a_{1}^{3}-6 a_{1} x^{2}+6 a_{1}^{2} x}{6\left(x-a_{1}\right)^{2}}, \quad B_{3}(1)=\frac{2 a_{2}^{3}-6 a_{2} x+2 x^{3}}{6\left(a_{2}-x\right)^{2}} .
\end{aligned}
$$

Theorem 2.7 Let $q>1, M>0,0<\alpha \leq 1,0 \leq a_{1}<a_{2}, h:\left[a_{1}, a_{2}\right] \rightarrow \mathbb{R}$ be an $\alpha$-fractional differentiable function and $D_{\alpha}(h) \in L_{\alpha}^{1}\left(\left[a_{1}, a_{2}\right]\right)$. Then the inequality

$$
\begin{aligned}
\mid h(x) & -\frac{\alpha}{a_{2}^{\alpha}-a_{1}^{\alpha}} \int_{a_{1}}^{a_{2}} h(s) d_{\alpha} s \mid \\
\leq & M \frac{x-a_{1}}{a_{2}^{\alpha}-a_{1}^{\alpha}}\left(A_{1}(\alpha)\right)^{1-1 / q}\left(\frac{-8 a_{1}^{\alpha}+2 a_{1}^{\alpha-1} x+2 x^{\alpha-1} a_{1}+4 x^{\alpha}}{12}\right)^{1 / q} \\
& +M \frac{a_{2}-x}{a_{2}^{\alpha}-a_{1}^{\alpha}}\left(B_{1}(\alpha)\right)^{1-1 / q}\left(\frac{a_{2}^{\alpha}-x^{\alpha}}{2}\right)^{1 / q}
\end{aligned}
$$

holds if $\left|h^{\prime}\right|^{q}$ is convex on $\left[a_{1}, a_{2}\right]$ and $\left|h^{\prime}(x)\right|^{q} \leq M$, where

$$
A_{1}(\alpha)=\frac{2 a_{1}^{\alpha}+a_{1}^{\alpha-1} x+x^{\alpha-1} a_{1}+2 x^{\alpha}-6 a_{1}^{\alpha}}{6}, \quad B_{1}(\alpha)=\frac{a_{2}^{\alpha}-x^{\alpha}}{2} .
$$

Proof It follows from the proof of Theorem 2.2 that

$$
\begin{aligned}
\mid h(x) & -\frac{\alpha}{a_{2}^{\alpha}-a_{1}^{\alpha}} \int_{a_{1}}^{a_{2}} h(s) d_{\alpha} s \mid \\
\leq & \frac{x-a_{1}}{a_{2}^{\alpha}-a_{1}^{\alpha}} \int_{0}^{1}\left((1-t) a_{1}^{\alpha-1}+t x^{\alpha-1}\right)\left(\left((1-t) a_{1}+t x\right)-a_{1}^{\alpha}\right)\left|h^{\prime}\left((1-t) a_{1}+t x\right)\right| d t \\
& +\frac{a_{2}-x}{a_{2}^{\alpha}-a_{1}^{\alpha}} \int_{0}^{1}\left(a_{2}^{\alpha}-\left((1-t) a_{2}^{\alpha}+t x^{\alpha}\right)\right)\left|h^{\prime}\left((1-t) a_{2}+t x\right)\right| d t .
\end{aligned}
$$

From the power-mean inequality and convexity of $\left|h^{\prime}\right|^{q}$ together with the identities

$$
\int_{0}^{1}\left((1-t) a_{1}^{\alpha-1}+t x^{\alpha-1}\right)\left(\left((1-t) a_{1}+t x\right)-a_{1}^{\alpha}\right) d t=\frac{2 a_{1}^{\alpha}+a_{1}^{\alpha-1} x+x^{\alpha-1} a_{1}+2 x^{\alpha}-6 a_{1}^{\alpha}}{6}
$$

and

$$
\int_{0}^{1}\left(a_{2}^{\alpha}-\left((1-t) a_{2}^{\alpha}+t x^{\alpha}\right)\right) d t=\frac{a_{2}^{\alpha}-x^{\alpha}}{2}
$$

we get

$$
\begin{aligned}
& \int_{0}^{1}\left((1-t) a_{1}^{\alpha-1}+t x^{\alpha-1}\right)\left(\left((1-t) a_{1}+t x\right)-a_{1}^{\alpha}\right)\left|h^{\prime}\left((1-t) a_{1}+t x\right)\right| d t \\
& \leq\left(\int_{0}^{1}\left((1-t) a_{1}^{\alpha-1}+t x^{\alpha-1}\right)\left(\left((1-t) a_{1}+t x\right)-a_{1}^{\alpha}\right) d t\right)^{1-1 / q} \\
& \quad \times\left(\int_{0}^{1}\left((1-t) a_{1}^{\alpha-1}+t x^{\alpha-1}\right)\left(\left((1-t) a_{1}+t x\right)-a_{1}^{\alpha}\right)\left|h^{\prime}\left((1-t) a_{1}+t x\right)\right|^{q} d t\right)^{1 / q}
\end{aligned}
$$




$$
\begin{aligned}
& \int_{0}^{1}\left(a_{2}^{\alpha}-\left((1-t) a_{2}^{\alpha}+t x^{\alpha}\right)\right)\left|h^{\prime}\left((1-t) a_{2}+t x\right)\right| d t \\
& \leq\left(\int_{0}^{1}\left(a_{2}^{\alpha}-\left((1-t) a_{2}^{\alpha}+t x^{\alpha}\right)\right) d t\right)^{1-1 / q} \\
& \times\left(\int_{0}^{1}\left(a_{2}^{\alpha}-\left((1-t) a_{2}^{\alpha}+t x^{\alpha}\right)\right)\left|h^{\prime}\left((1-t) a_{2}+t x\right)\right|^{q} d t\right)^{1 / q}, \\
& \int_{0}^{1}\left((1-t) a_{1}^{\alpha-1}+t x^{\alpha-1}\right)\left(\left((1-t) a_{1}+t x\right)-a_{1}^{\alpha}\right)\left|h^{\prime}\left((1-t) a_{1}+t x\right)\right|^{q} d t \\
& \leq \int_{0}^{1}\left((1-t) a_{1}^{\alpha-1}+t x^{\alpha-1}\right)\left(\left((1-t) a_{1}+t x\right)-a_{1}^{\alpha}\right)\left[(1-t)\left|h^{\prime}\left(a_{1}\right)\right|^{q}+t\left|h^{\prime}(x)\right|^{q}\right] d t \\
& =\left|h^{\prime}\left(a_{1}\right)\right|^{q} \int_{0}^{1}\left((1-t) a_{1}^{\alpha-1}+t x^{\alpha-1}\right)\left(\left((1-t) a_{1}+t x\right)-a_{1}^{\alpha}\right)(1-t) d t \\
& +\left|h^{\prime}(x)\right|^{q} \int_{0}^{1}\left((1-t) a_{1}^{\alpha-1}+t x^{\alpha-1}\right)\left(\left((1-t) a_{1}+t x\right)-a_{1}^{\alpha}\right) t d t \\
& =\left|h^{\prime}\left(a_{1}\right)\right|^{q}\left(\frac{1}{4} a_{1}^{\alpha}+\frac{1}{12} a_{1}^{\alpha-1} x+\frac{1}{12} x^{\alpha-1} a_{1}+\frac{1}{12} x^{\alpha}-\frac{1}{2} a_{1}^{\alpha}\right) \\
& +\left|h^{\prime}(x)\right|^{q}\left(\frac{1}{12} a_{1}^{\alpha}+\frac{1}{12} a_{1}^{\alpha-1} x+\frac{1}{12} x^{\alpha-1} a_{1}+\frac{1}{4} x^{\alpha}-\frac{1}{2} a_{1}^{\alpha}\right) \\
& \leq M^{q}\left(\frac{-8 a_{1}^{\alpha}+2 a_{1}^{\alpha-1} x+2 x^{\alpha-1} a_{1}+4 x^{\alpha}}{12}\right), \\
& \int_{0}^{1}\left(a_{2}^{\alpha}-\left((1-t) a_{2}^{\alpha}+t x^{\alpha}\right)\right)\left|h^{\prime}\left((1-t) a_{2}+t x\right)\right|^{q} d t \\
& \leq \int_{0}^{1}\left(a_{2}^{\alpha}-\left((1-t) a_{2}^{\alpha}+t x^{\alpha}\right)\right)\left[(1-t)\left|h^{\prime}\left(a_{2}\right)\right|^{q}+t\left|h^{\prime}(x)\right|^{q}\right] d t \\
& =\left|h^{\prime}\left(a_{2}\right)\right|^{q}\left(\frac{a_{2}^{\alpha}-x^{\alpha}}{6}\right)+\left|h^{\prime}(x)\right|^{q}\left(\frac{a_{2}^{\alpha}-x^{\alpha}}{3}\right) \\
& \leq M^{q}\left(\frac{a_{2}^{\alpha}-x^{\alpha}}{2}\right) \text {. }
\end{aligned}
$$

Therefore, Theorem 2.7 follows easily from (2.6)-(2.10).

Theorem 2.8 Let $q>1,0<\alpha \leq 1,0 \leq a_{1}<a_{2}, h:\left[a_{1}, a_{2}\right] \rightarrow \mathbb{R}$ be an $\alpha$-fractional differentiable function and $D_{\alpha}(h) \in L_{\alpha}^{1}\left(\left[a_{1}, a_{2}\right]\right)$. Then the inequality

$$
\begin{aligned}
& \left|h(x)-\frac{\alpha}{a_{2}^{\alpha}-a_{1}^{\alpha}} \int_{a_{1}}^{a_{2}} h(s) d_{\alpha} s\right| \\
& \quad \leq \frac{x-a_{1}}{a_{2}^{\alpha}-a_{1}^{\alpha}} A_{1}(\alpha)\left|h^{\prime}\left(\frac{C_{1}(\alpha)}{A_{1}(\alpha)}\right)\right|+\frac{a_{2}-x}{a_{2}^{\alpha}-a_{1}^{\alpha}} B_{1}(\alpha)\left|h^{\prime}\left(\frac{C_{2}(\alpha)}{B_{1}(\alpha)}\right)\right|
\end{aligned}
$$

holds if $\left|h^{\prime}\right|^{q}$ is concave on $\left[a_{1}, a_{2}\right]$, where

$$
\begin{aligned}
& A_{1}(\alpha)=\frac{x^{\alpha+1}-a_{1}^{\alpha+1}}{(\alpha+1)\left(x-a_{1}\right)}-a_{1}^{\alpha}, \\
& B_{1}(\alpha)=a_{2}^{\alpha}-\frac{x^{\alpha+1}-a_{2}^{\alpha+1}}{(\alpha+1)\left(a_{2}-x\right)},
\end{aligned}
$$




$$
\begin{aligned}
C_{1}(\alpha)= & \frac{x^{\alpha+2}-a_{1}^{\alpha+2}}{(\alpha+1)\left(x-a_{1}\right)}-\frac{x^{\alpha+3}+a_{1}^{\alpha+3}}{(\alpha+1)\left(x-a_{1}\right)^{2}(\alpha+2)} \\
& +\frac{a_{1} x}{(\alpha+1)\left(x-a_{1}\right)^{2}(\alpha+2)}\left(x^{\alpha+1}+a_{1}^{\alpha+1}\right)-a_{1}^{\alpha} \frac{\left(a_{1}+x\right)}{2}, \\
C_{2}(\alpha)= & a_{2}^{\alpha} \frac{\left(a_{2}+x\right)}{2}+\frac{a_{2}^{\alpha+3}+x^{\alpha+3}}{(\alpha+1)\left(a_{2}-x\right)^{2}(\alpha+2)} \\
& -\frac{a_{2} x}{(\alpha+1)\left(a_{2}-x\right)^{2}(\alpha+2)}\left(x^{\alpha+1}+a_{2}^{\alpha+1}\right)+\frac{a_{2}^{\alpha+2}-x^{\alpha+2}}{(\alpha+1)\left(a_{2}-x\right)} .
\end{aligned}
$$

Proof It is well known that $\left|h^{\prime}\right|$ is concave due to $\left|h^{\prime}\right|^{q}$ being concave. It follows from Lemma 2.1 that

$$
\begin{aligned}
\mid h(x) & -\frac{\alpha}{a_{2}^{\alpha}-a_{1}^{\alpha}} \int_{a_{1}}^{a_{2}} h(s) d_{\alpha} s \mid \\
\leq & \frac{x-a_{1}}{a_{2}^{\alpha}-a_{1}^{\alpha}} \int_{0}^{1}\left(\left((1-t) a_{1}+t x\right)^{\alpha}-a_{1}^{\alpha}\right)\left|h^{\prime}\left((1-t) a_{1}+t x\right)\right| d t \\
& +\frac{a_{2}-x}{a_{2}^{\alpha}-a_{1}^{\alpha}} \int_{0}^{1}\left(a_{2}^{\alpha}-\left((1-t) a_{2}+t x\right)^{\alpha}\right)\left|h^{\prime}\left((1-t) a_{2}+t x\right)\right| d t .
\end{aligned}
$$

Making use of Jensen's integral inequality, we have

$$
\begin{aligned}
& \int_{0}^{1}\left(\left((1-t) a_{1}+t x\right)^{\alpha}-a_{1}^{\alpha}\right)\left|h^{\prime}\left((1-t) a_{1}+t x\right)\right| d t \\
& \quad \leq \int_{0}^{1}\left(\left((1-t) a_{1}+t x\right)^{\alpha}-a_{1}^{\alpha}\right)\left|h^{\prime}\left(\frac{\int_{0}^{1}\left(\left((1-t) a_{1}+t x\right)^{\alpha}-a_{1}^{\alpha}\right)\left((1-t) a_{1}+t x\right) d t}{\int_{0}^{1}\left(\left((1-t) a_{1}+t x\right)^{\alpha}-a_{1}^{\alpha}\right) d t}\right)\right| d t \\
& \quad=A_{1}(\alpha)\left|h^{\prime}\left(\frac{C_{1}(\alpha)}{A_{1}(\alpha)}\right)\right|, \\
& \int_{0}^{1}\left(a_{2}^{\alpha}-\left((1-t) a_{2}+t x\right)^{\alpha}\right)\left|h^{\prime}\left((1-t) a_{2}+t x\right)\right| d t \\
& \quad \leq \int_{0}^{1}\left(a_{2}^{\alpha}-\left((1-t) a_{2}+t x\right)^{\alpha}\right)\left|h^{\prime}\left(\frac{\int_{0}^{1}\left(a_{2}^{\alpha}-\left((1-t) a_{2}+t x\right)^{\alpha}\right)\left((1-t) a_{2}+t x\right) d t}{\int_{0}^{1}\left(a_{2}^{\alpha}-\left((1-t) a_{2}+t x\right)^{\alpha}\right) d t}\right)\right| d t \\
& \quad=B_{1}(\alpha)\left|h^{\prime}\left(\frac{C_{2}(\alpha)}{B_{1}(\alpha)}\right)\right|,
\end{aligned}
$$

where we have used the identities

$$
\begin{aligned}
& \int_{0}^{1}\left(\left((1-t) a_{1}+t x\right)^{\alpha}-a_{1}^{\alpha}\right) d t=A_{1}(\alpha), \\
& \int_{0}^{1}\left(a_{2}^{\alpha}-\left((1-t) a_{2}+t x\right)^{\alpha}\right) d t=B_{1}(\alpha), \\
& \int_{0}^{1}\left(\left((1-t) a_{1}+t x\right)^{\alpha}-a_{1}^{\alpha}\right)\left((1-t) a_{1}+t x\right) d t=C_{1}(\alpha), \\
& \int_{0}^{1}\left(a_{2}^{\alpha}-\left((1-t) x+t a_{2}\right)^{\alpha}\right)\left((1-t) a_{2}+t x\right) d t=C_{2}(\alpha) .
\end{aligned}
$$


Remark 2.9 If $\alpha=1$, then Theorem 2.8 becomes

$$
\begin{aligned}
\mid h(x) & -\frac{\alpha}{a_{2}^{\alpha}-a_{1}^{\alpha}} \int_{a_{1}}^{a_{2}} h(s) d_{\alpha} s \mid \\
\leq & \frac{\left(x-a_{1}\right)^{2}}{2\left(a_{2}-a_{1}\right)}\left|h^{\prime}\left(\frac{2 x^{4}-5 a_{1} x^{3}+3 a_{1}^{2} x^{2}+x a_{1}^{3}-a_{1}^{4}}{3\left(x-a_{1}\right)}\right)\right| \\
& +\frac{\left(a_{2}-x\right)^{2}}{2\left(a_{2}-a_{1}\right)}\left|h^{\prime}\left(\frac{4 x^{4}-a_{2} x^{3}-3 x^{2} a_{2}^{2}-7 a_{2}^{3} x+7 a_{2}^{4}}{3\left(a_{2}-x\right)}\right)\right| .
\end{aligned}
$$

Theorem 2.10 Let $q>1,0<\alpha \leq 1,0 \leq a_{1}<a_{2}, h:\left[a_{1}, a_{2}\right] \rightarrow \mathbb{R}$ be an $\alpha$-fractional differentiable function and $D_{\alpha}(h) \in L_{\alpha}^{1}\left(\left[a_{1}, a_{2}\right]\right)$. Then the inequality

$$
\begin{aligned}
& \left|h(x)-\frac{\alpha}{a_{2}^{\alpha}-a_{1}^{\alpha}} \int_{a_{1}}^{a_{2}} h(s) d_{\alpha} s\right| \\
& \quad \leq \frac{x-a_{1}}{a_{2}^{\alpha}-a_{1}^{\alpha}} A_{1}(\alpha)\left|h^{\prime}\left(\frac{C_{1}(\alpha)}{A_{1}(\alpha)}\right)\right|+\frac{a_{2}-x}{a_{2}^{\alpha}-a_{1}^{\alpha}} B_{1}(\alpha)\left|h^{\prime}\left(\frac{C_{2}(\alpha)}{B_{1}(\alpha)}\right)\right|
\end{aligned}
$$

holds if $\left|h^{\prime}\right|^{q}$ is concave on $\left[a_{1}, a_{2}\right]$, where

$$
\begin{aligned}
& A_{1}(\alpha)=\frac{2 a_{1}^{\alpha}+a_{1}^{\alpha-1} x+x^{\alpha-1} a_{1}+2 x^{\alpha}-6 a_{1}^{\alpha}}{6}, \quad B_{1}(\alpha)=\frac{a_{2}^{\alpha}-x^{\alpha}}{2}, \\
& C_{1}(\alpha)=\frac{-3 a_{1}^{\alpha+1}+x+x^{\alpha-1} a_{1}^{\alpha+1}+x^{\alpha} a_{1}-5 x a_{1}^{\alpha}+x^{2} a_{1}^{\alpha-1}+x a_{1}+3 x^{\alpha+1}}{12} \\
& C_{2}(\alpha)=\frac{a_{2}^{\alpha+1}-x^{\alpha} a_{2}+2 x a_{2}^{\alpha}-2 x^{\alpha+1}}{6} .
\end{aligned}
$$

Proof From the concavity of $\left|h^{\prime}\right|^{q}$ we know that $\left|h^{\prime}\right|$ is also concave, then from Lemma 2.1 we have

$$
\begin{aligned}
& \left|h(x)-\frac{\alpha}{a_{2}^{\alpha}-a_{1}^{\alpha}} \int_{a_{1}}^{a_{2}} h(s) d_{\alpha} s\right| \\
& \leq \frac{x-a_{1}}{a_{2}^{\alpha}-a_{1}^{\alpha}} \int_{0}^{1}\left((1-t) a_{1}^{\alpha-1}+t x^{\alpha-1}\right)\left(\left((1-t) a_{1}+t x\right)-a_{1}^{\alpha}\right)\left|h^{\prime}\left((1-t) a_{1}+t x\right)\right| d t \\
& \quad+\frac{a_{2}-x}{a_{2}^{\alpha}-a_{1}^{\alpha}} \int_{0}^{1}\left(a_{2}^{\alpha}-\left((1-t) a_{2}^{\alpha}+t x^{\alpha}\right)\right)\left|h^{\prime}\left((1-t) a_{2}+t x\right)\right| d t
\end{aligned}
$$

It follows from the Jensen integral inequality that

$$
\begin{aligned}
& \int_{0}^{1}\left((1-t) a_{1}^{\alpha-1}+t x^{\alpha-1}\right)\left(\left((1-t) a_{1}+t x\right)-a_{1}^{\alpha}\right)\left|h^{\prime}\left((1-t) a_{1}+t x\right)\right| d t \\
& \quad \leq \int_{0}^{1}\left((1-t) a_{1}^{\alpha-1}+t x^{\alpha-1}\right)\left(\left((1-t) a_{1}+t x\right)-a_{1}^{\alpha}\right) \\
& \quad \times\left|h^{\prime}\left(\frac{\int_{0}^{1}\left((1-t) a_{1}^{\alpha-1}+t x^{\alpha-1}\right)\left(\left((1-t) a_{1}+t x\right)-a_{1}^{\alpha}\right)\left((1-t) a_{1}+t x\right) d t}{\int_{0}^{1}\left((1-t) a_{1}^{\alpha-1}+t x^{\alpha-1}\right)\left(\left((1-t) a_{1}+t x\right)-a_{1}^{\alpha}\right) d t}\right)\right| d t \\
& =A_{1}(\alpha) h^{\prime}\left(\frac{C_{1}(\alpha)}{A_{1}(\alpha)}\right)
\end{aligned}
$$




$$
\begin{aligned}
& \int_{0}^{1}\left(a_{2}^{\alpha}-\left((1-t) a_{2}^{\alpha}+t x^{\alpha}\right)\right)\left|h^{\prime}\left((1-t) a_{2}+t x\right)\right| d t \\
& \quad \leq \int_{0}^{1}\left(a_{2}^{\alpha}-\left((1-t) a_{2}^{\alpha}+t x^{\alpha}\right)\right)\left|h^{\prime}\left(\frac{\int_{0}^{1}\left(a_{2}^{\alpha}-\left((1-t) a_{2}^{\alpha}+t x^{\alpha}\right)\right)\left((1-t) a_{2}+t x\right) d t}{\int_{0}^{1}\left(a_{2}^{\alpha}-\left((1-t) a_{2}^{\alpha}+t x^{\alpha}\right)\right) d t}\right)\right| \\
& \quad=B_{1}(\alpha) h^{\prime}\left(\frac{C_{2}(\alpha)}{B_{1}(\alpha)}\right) .
\end{aligned}
$$

Remark 2.11 If $\alpha=1$, then Theorem 2.10 leads to

$$
\begin{aligned}
& \left|h(x)-\frac{1}{a_{2}-a_{1}} \int_{a_{1}}^{a_{2}} h(s) d s\right| \\
& \quad \leq \frac{\left(x-a_{1}\right)^{2}}{2\left(a_{2}-a_{1}\right)}\left|h^{\prime}\left(\frac{2 x^{2}-a_{1} x-a_{1}^{2}}{3\left(x-a_{1}\right)}\right)\right|+\frac{\left(a_{2}-x\right)^{2}}{2\left(a_{2}-a_{1}\right)}\left|h^{\prime}\left(\frac{a_{2}^{2}+a_{2} x-2 x^{2}}{3\left(a_{2}-x\right)}\right)\right| .
\end{aligned}
$$

Remark 2.12 If $\alpha=1$ and $x=\left(a_{1}+a_{2}\right) / 2$, then Theorem 2.10 becomes

$$
\left|h(x)-\frac{1}{a_{2}-a_{1}} \int_{a_{1}}^{a_{2}} h(s) d s\right| \leq \frac{a_{2}-a_{1}}{8}\left[\left|h^{\prime}\left(\frac{2 a_{1}+a_{2}}{3}\right)\right|+\left|h^{\prime}\left(\frac{a_{1}+2 a_{2}}{3}\right)\right|\right] .
$$

\section{Applications to means}

Let $a, b>0$ with $a \neq b$. Then the arithmetic mean $A(a, b)$, logarithmic mean $L(a, b)$ and generalized logarithmic mean $L_{(\alpha, r)}(a, b)$ of $a$ and $b$ are defined by

$$
A(a, b)=\frac{a+b}{2}, \quad L(a, b)=\frac{b-a}{\log b-\log a}, \quad L_{(\alpha, r)}(a, b)=\left[\frac{\alpha\left(b^{r+\alpha}-a^{r+\alpha}\right)}{(r+\alpha)\left(b^{\alpha}-a^{\alpha}\right)}\right]^{1 / r}
$$

respectively.

Recently, the bivariate means have been the subject of intensive research, many remarkable inequalities for the bivariate means can be found in the literature [25-60].

Let $h(x)=x^{r}$ and $h(x)=1 / x$. Then Corollary 2.3 immediately leads to Theorems 3.1 and 3.2.

Theorem 3.1 Let $r>1$ and $\alpha \in(0,1]$. Then the inequality

$$
\begin{aligned}
& \left|A^{r}\left(a_{1}, a_{2}\right)-L_{(\alpha, r)}^{r}\left(a_{1}, a_{2}\right)\right| \\
& \leq \frac{r\left(a_{2}-a_{1}\right)}{2\left(a_{2}^{\alpha}-a_{1}^{\alpha}\right)}\left[\left(\frac{2 a_{1}^{\alpha-1} a_{2}-10 a_{1}^{\alpha}+a_{1}+a_{2}}{24}\right)\left|a_{1}\right|^{r-1}+\frac{a_{1}}{12}\left(\frac{a_{1}+a_{2}}{2}\right)^{\alpha-1}\left|a_{1}\right|^{r-1}\right. \\
& \quad+\left(\frac{5 a_{1}+3 a_{2}-12 a_{1}^{\alpha}}{24}\right)\left|\frac{a_{1}+a_{2}}{2}\right|^{r-1}+\frac{a_{1}}{12}\left(\frac{a_{1}+a_{2}}{2}\right)^{\alpha-1}\left|\frac{a_{1}+a_{2}}{2}\right|^{r-1} \\
& \quad+\frac{1}{6} a_{2}^{\alpha}\left|a_{2}\right|^{r-1}-\frac{1}{6}\left(\frac{a_{1}+a_{2}}{2}\right)^{\alpha}\left|a_{2}\right|^{r-1}+\frac{a_{2}^{\alpha}}{3}\left|\frac{a_{1}+a_{2}}{2}\right|^{r-1} \\
& \left.\quad-\frac{1}{3}\left(\frac{a_{1}+a_{2}}{2}\right)^{\alpha}\left|\frac{a_{1}+a_{2}}{2}\right|^{r-1}\right]
\end{aligned}
$$

holds for all $a_{1}, a_{2}>0$. 
Theorem 3.2 Let $r>1$ and $\alpha \in(0,1]$. Then the inequality

$$
\begin{aligned}
& \left|A^{r}\left(a_{1}, a_{2}\right)-L_{(\alpha, r)}^{r}\left(a_{1}, a_{2}\right)\right| \\
& \leq \frac{\left(a_{2}-a_{1}\right)}{2\left(a_{2}^{\alpha}-a_{1}^{\alpha}\right)}\left[\left(\frac{2 a_{1}^{\alpha-1} b-10 a_{1}^{\alpha}+a_{1}+a_{2}}{24}\right)\left|a_{1}\right|^{-2}+\frac{a_{1}}{12}\left(\frac{a_{1}+a_{2}}{2}\right)^{\alpha-1}\left|a_{1}\right|^{-2}\right. \\
& \quad+\left(\frac{5 a_{1}+3 a_{2}-12 a_{1}^{\alpha}}{24}\right)\left|\frac{a_{1}+a_{2}}{2}\right|^{-2}+\frac{a_{1}}{12}\left(\frac{a_{1}+a_{2}}{2}\right)^{\alpha-1}\left|\frac{a_{1}+a_{2}}{2}\right|^{-2} \\
& \quad+\frac{1}{6} a_{2}^{\alpha}\left|a_{2}\right|^{-2}-\frac{1}{6}\left(\frac{a_{1}+a_{2}}{2}\right)^{\alpha}\left|a_{2}\right|^{-2}+\frac{a_{2}^{\alpha}}{3}\left|\frac{a_{1}+a_{2}}{2}\right|^{-2} \\
& \left.\quad-\frac{1}{3}\left(\frac{a_{1}+a_{2}}{2}\right)^{\alpha}\left|\frac{a_{1}+a_{2}}{2}\right|^{-2}\right]
\end{aligned}
$$

holds for all $a_{1}, a_{2}>0$.

\section{Results and discussion}

There are many results devoted to the well-known Ostrowski inequality. This inequality has many applications in the area of numerical analysis. In this paper, we give results for Ostrowski inequality containing conformable fractional integrals and their applications for means. First, we prove an identity associated with the Ostrowski inequality for conformable fractional integrals. By using this identity and convexity of different classes of functions and some well-known inequalities, we obtain several results for the inequality. The inequalities derived here are also pointed out to correspond to some known results, being special cases. At the end, we also present applications for means. The presented idea may stimulate further research in the theory of conformable fractional integrals.

\section{Conclusion}

In this paper, we prove an identity associated with the Ostrowski inequality for conformable fractional integral, present several Ostrowski type inequalities involving the conformable fractional integrals, and provide the applications in bivariate means theory. The idea and results presented are novel and interesting.

\section{Acknowledgements}

The research was supported by the Natural Science Foundation of China (Grants Nos. 61673169, 61374086, 11371125, 11401191), the Tianyuan Special Funds of the National Natural Science Foundation of China (Grant No. 11626101) and the Natural Science Foundation of the Department of Education of Zhejiang Province (Grant No. Y201635325).

\section{Competing interests}

The authors declare that they have no competing interests.

\section{Authors' contributions}

All authors contributed equally to the writing of this paper. All authors read and approved the final manuscript.

\section{Author details}

${ }^{1}$ College of Science, Hunan City University, Yiyang, China. ${ }^{2}$ Department of Mathematics, University of Peshawar,

Peshawar, Pakistan. ${ }^{3}$ Department of Mathematics, Huzhou University, Huzhou, China.

\section{Publisher's Note}

Springer Nature remains neutral with regard to jurisdictional claims in published maps and institutional affiliations. 


\section{References}

1. Ostrowski, A.: Über die Absolutabweichung einer differentiierbaren Funktion von ihrem Integralmittelwert. Comment. Math. Helv. 10(1), 226-227 (1937)

2. Dragomir, S.S.: The Ostrowski integral inequality for mappings of bounded variation. Bull. Aust. Math. Soc. 60(3) 495-508 (1999)

3. Cerone, P., Cheung, W.S., Dragomir, S.S.: On Ostrowski type inequalities for Stieltjes intergals with absolutely continuous integrands and integrators of bounded variation. Comput. Math. Appl. 54(2), 183-191 (2007)

4. Dragomir, S.S.: Refinements of the generalised trapezoid and Ostrowski inequalities for functions of bounded variation. Arch. Math. 91(5), 450-460 (2008)

5. Alomari, M., Darus, M., Dragomir, S.S., Cerone, P.: Ostrowski type inequalities for functions whose derivatives are s-convex in the second sense. Appl. Math. Lett. 23(9), 1071-1076 (2010)

6. Alomari, M.W., Latif, M.A.: A weighted companion for the Ostrowski and the generalized trapezoid inequalities for mappings of bounded variation (2011). https://www.researchgate.net/publication/265179542

7. Alomari, M.W.: A generalization of weighted companion of Ostrowski integral inequality for mappings of bounded variation (2011). https://www.researchgate.net/publication/228451771

8. Tseng, K.-L.: Improvements of the Ostrowski integral inequality for mappings of bounded variation II. Appl. Math. Comput. 218(10), 5841-5847 (2012)

9. Alomari, M.W.: A companion of Dragomir's generalization of the Ostrowski inequality and applications to numerical integration. Ukr. Math. J. 64(4), 491-510 (2012)

10. Dragomir, S.S.: A companion of Ostrowski's inequality for functions of bounded variation and applications. Int. J. Nonlinear Anal. Appl. 5(1), 89-97 (2014)

11. Budak, H., Sarikaya, M.Z.: A new generalization of Ostrowski type inequality for mappings of bounded variation (2015). https://www.researchgate.net/publication/279874744

12. Budak, H., Sarikaya, M.Z.: New weighted Ostrowski type inequalities for mappings with first derivatives of bounded variation. Transylv. J. Math. Mech. 8(1), 21-27 (2016)

13. Qayyum, A., Shoaib, M., Faye, I.: A companion of Ostrowski type integral inequality using 5-step kernel with some applications. Filomat 30(13), 3601-3614 (2016)

14. Chu, Y.-M., Adil Khan, M., Khan, T.U., Ali, T.: Generalizations of Hermite-Hadamard type inequalities for MT-convex functions. J. Nonlinear Sci. Appl. 9(6), 4305-4316 (2016)

15. Wang, M.-K., Chu, Y.-M.: Landen inequalities for a class of hypergeometric functions with applications. Math. Inequal. Appl. 21(2), 521-537 (2018)

16. Adil Khan, M., Chu, Y.-M., Khan, T.U., Khan, J.: Some new inequalities of Hermite-Hadamard type for s-convex functions with applications. Open Math. 15, 1414-1430 (2017)

17. Yang, Z.-H., Zhang, W., Chu, Y.-M.: Sharp Gautschi inequality for parameter $0<p<1$ with applications. Math. Inequal. Appl. 20(4), 1107-1120 (2017)

18. Yang, Z.-H., Qian, W.-M., Chu, Y.-M.: On rational bounds for the gamma function. J. Inequal. Appl. 2017, Article ID 210 (2017)

19. Yang, Z.-H., Chu, Y.-M.: A monotonicity property involving the generalized elliptic integral of the first kind. Math. Inequal. Appl. 20(3), 729-735 (2017)

20. Yang, Z.-H., Qian, W.-M., Chu, Y.-M., Zhang, W.: Monotonicity rule for the quotient of two functions and its applications. J. Inequal. Appl. 2017, Article ID 106 (2017)

21. Yang, Z.-H., Qian, W.-M., Chu, Y.-M., Zhang, W.: On approximating the arithmetic-geometric mean and complete elliptic of the first kind. J. Math. Anal. Appl. 462(2), 1714-1726 (2018)

22. Budak, H., Sarikaya, M.Z.: On generalization of weighted Ostrowski type inequalities for functions of bounded variation. Asian-Eur. J. Math. (2018, to appear). https://doi.org/10.1142/S1793557118500493

23. Wang, M.-K., Li, Y.-M., Chu, Y.-M.: Inequalities and infinite product formula for Ramanujan generalized modular equation function. Ramanujan J. (2018, to appear). https://doi.org/10.1007/s11139-017-9888-3

24. Khalil, R., Al Horani, M., Yousef, A., Sababheh, M.: A new definition of fractional derivative. J. Comput. Appl. Math. 264, 65-70 (2014)

25. Chu, Y.-M., Zhang, X.-M.: Necessary and sufficient conditions such that extended mean values are Schur-convex or Schur-concave. J. Math. Kyoto Univ. 48(1), 229-238 (2008)

26. Chu, Y.-M., Zhang, X.-M., Wang, G.-D.: The Schur geometrical convexity of the extended mean values. J. Convex Anal. 15(4), 707-718 (2008)

27. Shi, M.-Y., Chu, Y.-M., Jiang, Y.-P.: Optimal inequalities among various means of two arguments. Abstr. Appl. Anal. 2009 Article ID 694394 (2009)

28. Wang, M.-K., Qiu, S.-L., Chu, Y.-M.: Infinite series formula for Hübner upper bound function with applications to Hersch-Pfluger distortion function. Math. Inequal. Appl. 21(3), 629-648 (2018)

29. Zhang, X.-H., Wang, G.-D., Chu, Y.-M.: Convex with respect to Hölder mean involving zero-balanced hypergeometric functions. J. Math. Anal. Appl. 353(1), 256-259 (2009)

30. Chu, Y.-M., Xia, W.-F.: Two sharp inequalities for power mean, geometric mean, and harmonic mean. J. Inequal. Appl. 2009, Article ID 741923 (2009)

31. Chu, Y.-M., Xia, W.-F.: Two optimal double inequalities between power mean and logarithmic mean. Comput. Math Appl. 60(1), 83-89(2010)

32. Xia, W.-F., Chu, Y.-M., Wang, G.-D.: The optimal upper and lower power mean bounds for a convex combination of the arithmetic and logarithmic means. Abstr. Appl. Anal. 2010, Article ID 604804 (2010)

33. Chu, Y.-M., Qiu, Y.-F., Wang, M.-K.: Sharp power mean bounds for the combination of Seiffert and geometric means. Abstr. Appl. Anal. 2010, Article ID 108920 (2010)

34. Chu, Y.-M., Qiu, Y.-F., Wang, M.-K., Wang, G.-D.: The optimal convex combination bounds of arithmetic and harmonic means for the Seiffert's mean. J. Inequal. Appl. 2010, Article ID 436457 (2010)

35. Chu, Y.-M., Wang, M.-K., Qiu, Y.-F.: An optimal double inequality between power-type Heron and Seiffert means. J. Inequal. Appl. 2010, Article ID 146945 (2010)

36. Wang, M.-K., Qiu, Y.-F., Chu, Y.-M.: Sharp bounds for Seiffert means in terms of Lehmer means. J. Math. Inequal. 4(4), 581-586 (2010) 
37. Chu, Y.-M., Long, B.-Y.: Best possible inequalities between generalized logarithmic mean and classical means. Abstr. Appl. Anal. 2010, Article ID 303286 (2010)

38. Long, B.-Y., Chu, Y.-M.: Optimal inequalities for generalized logarithmic, arithmetic, and geometric means. J. Inequal. Appl. 2010, Article ID 806825 (2010)

39. Wang, M.-K., Chu, Y.-M., Qiu, Y.-F., Qiu, S.-L.: An optimal power mean inequality for the complete elliptic integrals. Appl. Math. Lett. 24(6), 887-890 (2011)

40. Chu, Y.-M., Zong, C., Wang, G.-D.: Optimal convex combination bounds of Seiffert and geometric means for the arithmetic mean. J. Math. Inequal. 5(3), 429-434 (2011)

41. Chu, Y.-M., Wang, M.-K., Qiu, S.-L.: Sharp generalized Seiffert mean bounds for Toader mean. Abstr. Appl. Anal. 2011, Article ID 605259 (2011)

42. Chu, Y.-M., Wang, M.-K., Gong, W.-M.: Two sharp double inequalities for Seiffert mean. J. Inequal. Appl. 2011, Article ID $44(2011)$

43. Qiu, Y.-F., Wang, M.-K., Chu, Y.-M., Wang, G.-D.: Two sharp inequalities for Lehmer mean, identric mean and logarithmic mean. J. Math. Inequal. 5(3), 301-306 (2011)

44. Chu, Y.-M., Wang, M.-K., Wang, Z.-K.: Best possible inequalities among harmonic, geometric, logarithmic and Seiffert means. Math. Inequal. Appl. 15(2), 415-422 (2012)

45. Chu, Y.-M., Hou, S.-W.: Sharp bounds for Seiffert mean in terms of contraharmonic mean. Abstr. Appl. Anal. 2012, Article ID 425175 (2012)

46. Wang, M.-K., Wang, Z.-K., Chu, Y.-M.: An optimal double inequality between geometric and identric means. Appl. Math. Lett. 25(3), 471-475 (2012)

47. Chu, Y.-M., Wang, M.-K., Qiu, S.-L.: Optimal combinations bounds of root-square and arithmetic means for Toader mean. Proc. Indian Acad. Sci. Math. Sci. 122(1), 41-51 (2012)

48. Li, Y.-M., Long, B.-Y., Chu, Y.-M.:. Sharp bounds for the Neuman-Sándor mean in terms of generalized logarithmic mean. J. Math. Inequal. 6(4), 567-577 (2012)

49. Wang, M.-K., Chu, Y.-M., Qiu, S.-L., Jiang, Y.-P.: Convexity of the complete elliptic integrals of the first kind with respect to Hölder means. J. Math. Anal. Appl. 388(2), 1141-1146 (2012)

50. Zhao, T.-H., Chu, Y.-M., Liu, B.-Y.: Optimal bounds for Neuman-Sándor mean in terms of the convex combinations of harmonic, geometric, quadratic, and contraharmonic means. Abstr. Appl. Anal. 2012, Article ID 302635 (2012)

51. Chu, Y.-M., Wang, M.-K.: Optimal Lehmer mean bounds for the Toader mean. Results Math. 61(3-4), 223-229 (2012)

52. Wang, M.-K., Wang, Z.-K., Chu, Y.-M.: Inequalities between arithmetic-geometric, Gini, and Toader means. Abstr. Appl. Anal. 2012, Article ID 830585 (2012)

53. Chu, Y.-M., Long, B.-Y., Gong, W.-M., Song, Y.-Q.: Sharp bounds for Seiffert and Neuman-Sándor means in terms of generalized logarithmic means. J. Inequal. Appl. 2013, Article ID 10 (2013)

54. Chu, Y.-M., Wang, M.-K., Ma, X.-Y.: Sharp bounds for Toader mean in terms of contraharmonic mean with applications. J. Math. Inequal. 7(2), 161-166 (2013)

55. Chu, Y.-M., Long, B.-Y.: Bounds of the Neuman-Sándor mean using power and identric means. Abstr. Appl. Anal. 2013, Article ID 832591 (2013)

56. Yang, Z.-H., Qian, W.-M., Chu, Y.-M., Zhang, W.: On approximating the error function. Math. Inequal. Appl. 21(2), 469-479 (2018)

57. Wang, M.-K., Chu, Y.-M., Qiu, S.-L.: Sharp bounds for generalized elliptic integrals of the first kind. J. Math. Anal. Appl. 429(2), 744-757 (2015)

58. Wang, M.-K., Chu, Y.-M., Song, Y.-Q.: Asymptotical formulas for Gaussian and generalized hypergeometric functions. Appl. Math. Comput. 276, 44-60 (2016)

59. Wang, M.-K., Chu, Y.-M., Jiang, Y.-P.: Ramanujan's cubic transformation inequalities for zero-balanced hypergeometric functions. Rocky Mt. J. Math. 46(2), 679-691 (2016)

60. Qian, W.-M., Chu, Y.-M.: Sharp bounds for a special quasi-arithmetic mean in terms of arithmetic and geometric means with two parameters. J. Inequal. Appl. 2017, Article ID 274 (2017)

\section{Submit your manuscript to a SpringerOpen ${ }^{\circ}$ journal and benefit from:}

- Convenient online submission

- Rigorous peer review

- Open access: articles freely available online

- High visibility within the field

Retaining the copyright to your article

Submit your next manuscript at $\downarrow$ springeropen.com 